\title{
Effects of Sex Hormones on Nociception and the Analgesic Action of NSAIDs
}

\author{
Ji Yong Park, M.D., Hee Chul Han, M.D.*, and Seong Ho Chang, M.D. \\ Departments of Anesthesiology and Pain Medicine, and *Physiology \\ College of Medicine, Korea University, Seoul, Korea
}

$=$ Abstract $=$

Background: The effects of sex hormones on nociception and the analgesic actions of non-steroidal anti-inflammatory drugs (NSAIDs) in an acute arthritic pain model were investigated.

Methods: Rats were ovariectomized and randomly assigned to three experimental groups. The estrogen group $(n=45)$ received a $0.25 \mathrm{mg}$ pellet of $17 \beta$-estradiol, the placebo group $(n=45)$ received a $0.25 \mathrm{mg}$ pellet of a placebo and the progesterone group $(\mathrm{n}=45)$ received a $25 \mathrm{mg}$ pellet of progesterone. Arthritis was induced by injecting $2 \%$ carrageenan into the knee joint cavity of the right hind leg. Before and after the injection, rats were allowed to walk freely through a weight load apparatus. The weight load and the weight of the rat were measured for each test. One hour after injection, ibuprofen or NS-398, dissolved in dimethyl sulfoxide, was injected intraperitoneally $(1 \mathrm{mg} / \mathrm{kg} / \mathrm{ml})$.

Results: The carrageenan injection into the knee joint cavity of the right hind leg of the rat resulted in a significant decrease in the weight load on the injected leg. Estrogen-treated rats showed lower weight load reduction than the placebo and progesterone groups, NS-398 increased the weight load compared to rats not receiving NSAIDs.

Conclusions: These results suggest that the nociceptive response after acute inflammation was reduced by estrogen, and that only NS-398 had a good analgesic effect in the placebo and progesterone groups. It is likely that the analgesic effect of NSAIDs on the estrogen group was unremarkable compared to those of the placebo and progesterone groups because of the antinociceptive action of estrogen. (Korean J Anesthesiol 2003; 44: S 20 S 27)

Key Words: Anti-inflammatory agents, non-steroidal; estrogens; progesterone; prostaglandin-endoperoxide synthase.

\section{INTRODUCTION}

Pain does not discriminate. It affects both males and females, from children to the elderly, crossing ethnic lines

\footnotetext{
Received : January 6, 2003

Corresponding to : Seong Ho Chang, Department of Anesthesiology and Pain Medicine, Anam Hospital, 126-1 Anam-dong 5ga, Seongbuk-gu, Seoul, 136-705, Korea Tel: 02-920-5771, Fax: 02-929-2936

E-mail: torchid@korea.ac.kr

Thesis for the Degree of Master (Doctor).
}

and cultural boundaries. However as far as perception of pain is concerned, it is not universal. Population based pain research has shown that women are more likely than men to experience a variety of temporary and persistent pains and to report more severe pain, more frequent pain and pain of longer duration than men. Furthermore, epidemiological research suggests gender differences in the distribution of various clinical pain syndromes. Women have shown a higher prevalence of migraine and nonmigrainous headache, temporomandibular disorders, back pain and arthritis. ${ }^{1)}$ 
This gender differential reporting of pain may be a function of biological, cultural and psychological differences, divergent social role expectations, situational factors and an individual's past history. ${ }^{2)}$ Particularly one of the most obvious and frequently noted distinctions between females and males offered as an explanation for sex-related influences on pain and analgesia may be the contribution of sex hormones. However, there is very little agreement that sex hormones have pain modulatory effects. Sex hormones demonstrated influences on analgesia in some studies, ${ }^{3,4)}$ but not in others. ${ }^{5}$

Sex hormones also appear to influence analgesic responses to pharmacological agents. Some studies demonstrated increased sensitivity to opioids among male compared to female animals. ${ }^{6-8)}$ In contrast to these findings, another study showed no differences in gender responses to morphine. ${ }^{9)}$ Responses to nonopioid agents have also been examined, with males demonstrating greater cocaine-induced analgesia and females showing enhanced analgesic responses to nicotine. ${ }^{10)}$ Females also have demonstrated greater analgesic responses to cholinergic agonists. ${ }^{11)}$ Thus, most, but not all studies indicate enhanced opioid antinociception in male animals, while some other agents produce more robust analgesia for females. However few attempts have been made to investigate influences of sex hormones on non-steroidal anti-inflammatory drugs (NSAIDs).

Therefore in this study, effects of sex hormones on nociception and the analgesic actions of NSAIDs in an acute arthritic pain model were investigated.

\section{METHODS}

\section{Animals and preparation}

Female Sprague-Dawley rats (Sam:TacN (SD)BR, 120 $-150 \mathrm{~g}, 6$ weeks old at arrival; purchased from Samtako CO., Osan, Korea) were housed in groups of five in a temperature-controlled room $\left(22-25^{\circ} \mathrm{C}\right)$ illuminated from 7 A.M. to 7 P.M.. Food and water were available ad libitum.

Rats were anesthetized with a mixture of enflurane and oxygen. Bilateral ovariectomies were performed via a mid-ventral incision with ligation of the fallopian tubes just proximal to the ovaries and removal of the ovaries with surrounding fat. The peritoneum was sutured with a synthetic absorbable suture. The skin was sutured with a stapler.

One week after the ovariectomies, the rats were randomly assigned to three experimental groups. The first group (estrogen group, $\mathrm{n}=45$ ) received a $0.25 \mathrm{mg}$ pellet of $17 \beta$-estradiol, the second group (placebo group, $\mathrm{n}=$ 45) received a $0.25 \mathrm{mg}$ pellet of a placebo for $17 \beta$ estradiol and the third group (progesterone group, $n=45$ ) received a $25 \mathrm{mg}$ pellet of progesterone. All pellets were produced by Innovative Research of America (IRA, Sarasota, USA) and were implanted subcutaneously to the dorsum of the neck. The carrier material in the pellets was cholesterol, lactose, celluloses, phosphates and stearates. The pellets were reported by IRA to release their hormone at a constant rate for a period of 3 weeks. On the ninth day after pellet implantation, to induce inflammation in the rats, $50 \mu 1$ of $2 \% \lambda$-carrageenan suspended in sterile saline was injected into the knee joint cavity of the right hind leg of the rats under enflurane anesthesia (0.5-2\%).

Each group was classified into three subgroups in accordance with NSAIDs injected. One hour after the carrageenan injection, two subgroups received either ibuprofen (Biomol Research Laboratories, Inc., USA) or NS-398 (Biomol Research Laboratories, Inc., USA) dissolved in dimethyl sulfoxide (Sigma Chemical Co., USA) intraperitoneally $(1 \mathrm{mg} / \mathrm{kg} / \mathrm{ml})$ and for the third subgroup nothing was given.

\section{Behavior test}

A weight load test was performed as described by Min et al. $^{12)}$

This apparatus was composed of a starting box, a path and an arrival box (Fig. 1). The starting and arrival boxes were made with translucent and black acrylic plates, respectively. The starting box was illuminated with bright light. This setting made rats in the starting box move voluntarily toward the arrival box without stopping or turning around. The path of the apparatus was constructed in such a way that, while a rat was walking through it, 


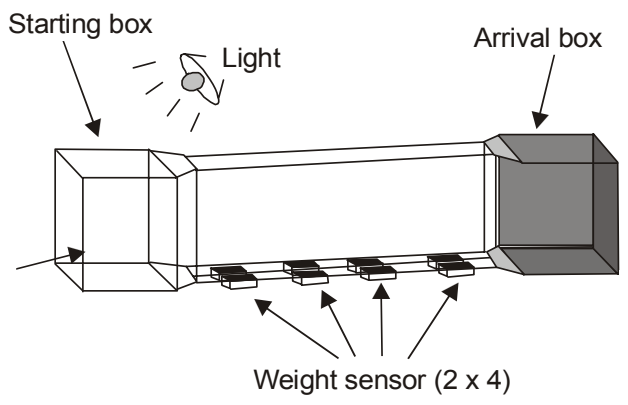

Fig. 1. Schematic diagram of the test apparatus. Test apparatus was composed of a starting box, a path and an arrival box. The starting and arrival boxes were made with translucent and black acrylic plates, respectively. The starting box was illuminated with bright light. This setting made rats in the starting box move voluntarily toward the arrival box without stopping or turning around. The path of the apparatus was constructed in such a way that, while a rat was walking through it, the weight load on a given leg of the rat could be monitored at a maximum of four different spots along the path. The floor of the path consisted of eight acrylic plates attached to load cells. These plates were lined up in two rows of four.

the weight load on a given leg of the rat could be monitored at a maximum of four different spots along the path. The floor of the path consisted of eight acrylic plates (width $\times$ length: $5 \times 10 \mathrm{~cm}$ ) attached to load cells (Dana load cell, strain gauge type, working range 0-1,000 g, Dana load cell LTD., Korea). These plates were lined up in two rows of four. The sidewalls of the path were built with two movable acrylic plates so that the width of the path could be adjusted according to the size and walking pattern of the rat. This adjustment prevented the rat from stepping on the plates of the contralateral side. The output of each load cell was fed to a digital amplifier (Cyber Amp 380, Axon Instruments, Inc., USA) for appropriate amplification and filtering. The processed signal was sent to a personal computer via an analog- digital converter (1401 Plus, UK) and plotted as a time-weight load curve using software (Spike 2, UK). Calibration of the weight sensor was performed by placing different weights on each load cell attached plate. Linear responses were obtained in the range of 10-300 g.

This test was initiated by placing the rat in the starting box. While the rat was walking through the path between the starting and arrival boxes, the investigator identified the plates on which the rat placed the hind paw of interest. Output signals from the load cells attached to these plates were selected for plotting time-weight load curves. The signals generated by improper stepping (i.e., stepping on the borderline between plates) were discarded. The test was repeated two or three times until five time-weight load curves were obtained for a given leg. The weight load tests and measurement of body weight were performed before and $6,9,12,24,60$ and 120 hours after the injection of carrageenan.

The measured values of weight load were normalized. The weight load value measured at a given time was expressed in percent of body weight.

\section{Statistics}

A repeated-measures ANOVA test was used to compare the variance among data from different experimental groups at a corresponding time point. When the repeatedmeasures ANOVA test indicated significance $(\mathrm{P}<0.05)$, post-hoc comparison tests were further performed to identify the source (s) of the variance.

\section{RESULTS}

The carrageenan injection into the knee joint cavity of the right hind leg of the rat resulted in a decrease in the weight load on the injected leg.

Comparing the antinociceptional effect of sex hormones only in rats not receiving NSAIDs, the weight loads of rats in the estrogen group decreased less than in the placebo and progesterone groups at 6, 9, 12, 24 and 60 hours after injection of carrageenan $(\mathrm{P}<0.05)$ (Fig. 2).

The analgesic effects of NSAIDs in the placebo group showed that NS-398 increased the weight load more than rats not receiving NSAIDs at 6,9 and 12 hours after injection of carrageenan $(\mathrm{P}<0.05)$. The ibuprofen also increased the weight load, although there were no significant differences (Fig. 3). The estrogen group showed that there were no differences to weight load increases in accordance with NSAIDs (Fig. 4). The progesterone group 


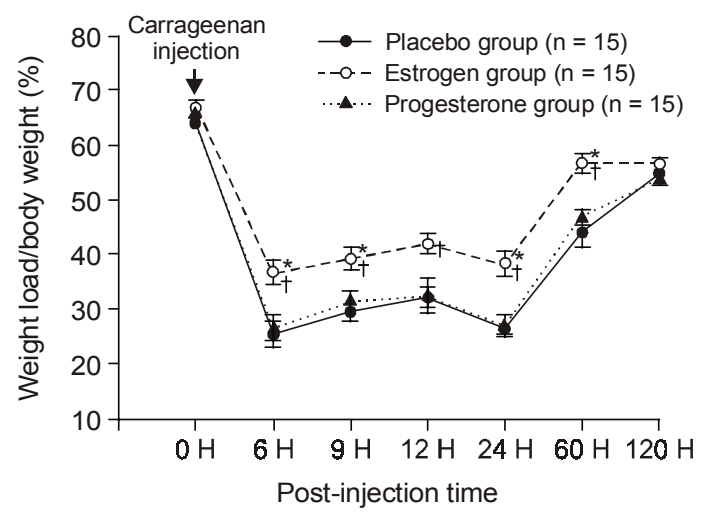

Fig. 2. Changes in weight load under sex hormonal influences after injection of carrageenan $(0 \mathrm{H})$. The ratio of weight load to body weight (mean \pm SEM) is plotted against time. The carrageenan injection into the knee joint cavity of the right hind leg of the rat resulted in a decrease of the weight load on the injected leg. The weight loads of rats in the estrogen group decreased less than in the placebo and progesterone groups at $6,9,12,24$ and 60 hours after injection of carrageenan $(\mathrm{P}<0.05)$. *: $\mathrm{P}<$ 0.05 , compared to placebo group, ${ }^{\dagger}: \mathrm{P}<0.05$, compared to progesterone group; $\mathrm{H}$, hour.

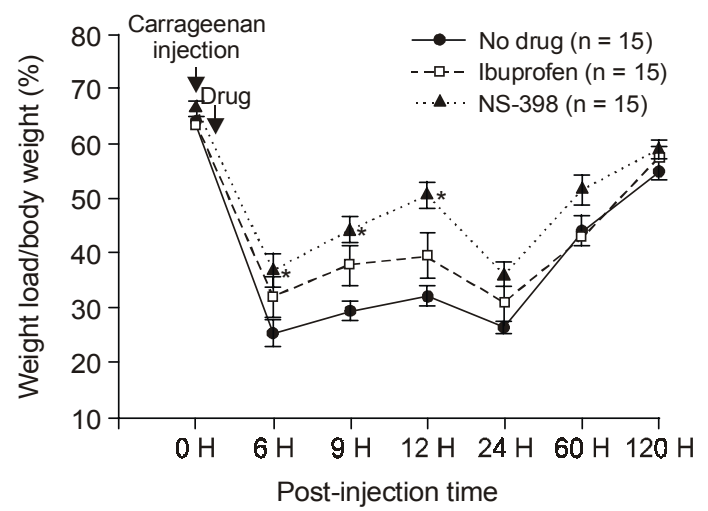

Fig. 3. Effects of an intraperitoneal injection of ibuprofen and NS-398 on the change in the weight loads induced by a carrageenan injection into the knee joint cavity in the placebo group. The ratio of weight load to body weight (mean \pm SEM) is plotted against time. This figure shows that NS-398 increased weight load more than rats receiving no drug at 6,9 and 12 hours after injection of carrageenan $(\mathrm{P}<0.05)$ and ibuprofen also increased the weight load, although there were no significant differences. *: $\mathrm{P}<0.05$, compared to no drug; $\mathrm{H}$, hour.

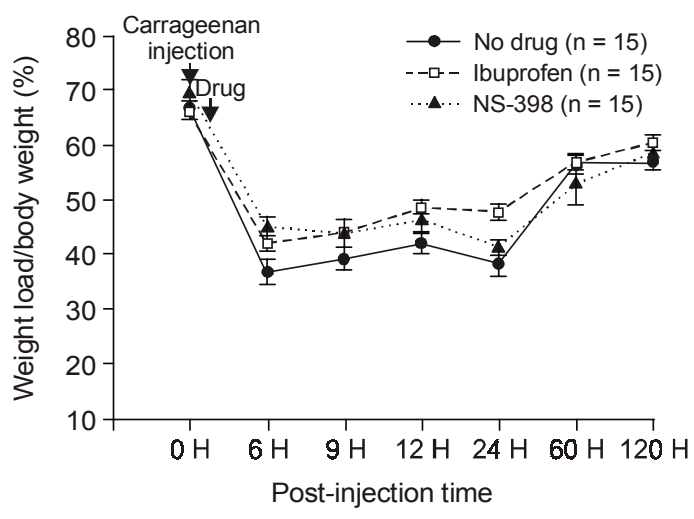

Fig. 4. Effects of an intraperitoneal injection of ibuprofen and NS-398 on the change in the weight loads induced by a carrageenan injection into the knee joint cavity in the estrogen group. The ratio of weight load to body weight (mean \pm SEM) is plotted against time. This figure shows that there were no differences to weight load increases in accordance with NSAIDs. H, hour.

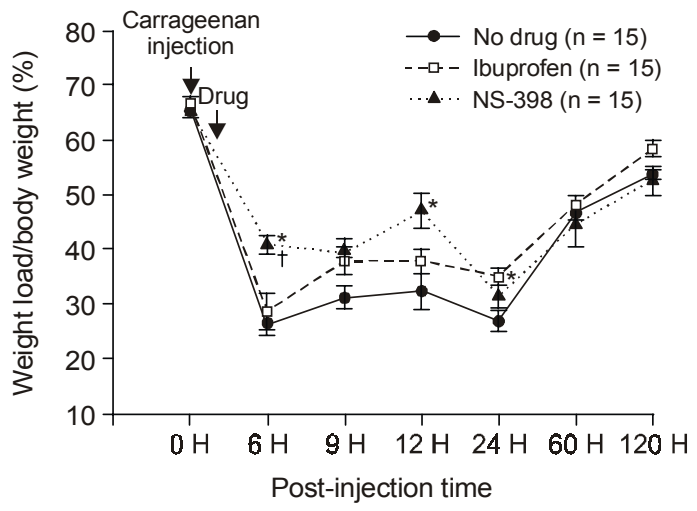

Fig. 5. Effects of an intraperitoneal injection of ibuprofen and NS-398 on the change in the weight loads induced by a carrageenan injection into the knee joint cavity in the progesterone group. The ratio of weight load to body weight (mean \pm SEM) is plotted against time. This figure shows that NS-398 increased the weight load more than rats receiving no drug at 6,12 and 24 hours after injection of carrageenan $(\mathrm{P}<0.05)$ and ibuprofen also increased the weight load, although there were no significant differences. *: $\mathrm{P}<0.05$, compared to no drug, ${ }^{+}: \mathrm{P}<$ 0.05 , compared to ibuprofen; $\mathrm{H}$, hour. 
showed that NS-398 increased the weight load more than rats not receiving NSAIDs at 6, 12 and 24 hours after injection of carrageenan $(\mathrm{P}<0.05)$ and ibuprofen also increased the weight load, although there were no significant differences (Fig. 5).

\section{DISCUSSION}

It was found from the results that a nociceptive response after acute inflammation was reduced by estrogen, and NS-398 only showed a good analgesic effect in the placebo and progesterone groups.

Sex hormones naturally fluctuate across the female menstrual cycle. The analog to the human menstrual cycle in rats is the estrous cycle, which is typically classified into four stages: metestrus, during which progeslevated while estrogen and LH levels are low; diestrus, characterized by relatively high levels of $\mathrm{LH}$, increasing estrogen and decreasing progesterone; proestrus, during which estrogen, progesterone, LH and FSH peak; and estrus, when females are sexually receptive and estrogen, progesterone, LH and FSH all reach low levels. ${ }^{13-15)}$

Studies examining responses to experimentally induced pain in female rats at different points in the estrous cycle have produced inconsistent results. This is likely due to methodological differences across studies. The nociceptive assays used vary widely in both the painful stimulus and the measured responses. Regarding the former, some nociceptive procedures produce primarily cutaneous stimulation (e.g. electrical, thermal), while others stimulate deeper structures (e.g. mechanical, chemical). The nociceptive responses assessed also differ across assays. Some assays involve assessment of motor or autonomic reflexes, which are typically thought to be produced at the level of the spinal cord or brainstem. Other assays involve assessment of the threshold for a specific behavior (e.g. footlick, escape), while others require determining the frequency of more complex behaviors over time (e.g. writhing, behavioral crises). In addition to this variability in nociceptive responses, the light-dark cycle and the precise timing of assessment (e.g. early vs. late proestrus) are important variables.
In the results of studies examining hormonal influences on nociceptive responses, some studies report greater pain sensitivity during metestrus and diestrus. ${ }^{16-19)}$ However, other studies suggest that pain sensitivity peaks during late proestrus and early estrus compared to metestrus and diestrus. $^{20-24)}$ It is notable that two of the studies demonstrating enhanced sensitivity during metestrus used visceral nociceptive stimuli. ${ }^{16,18)}$ Bradshaw et al. ${ }^{16)}$ examined escape responses to vaginal and uterine distention, which involved having the rat perform a previously learned behavior of extending her head into a tube. Rats performed escape responses to similar pressures resulting from both uterine and vaginal stimulation at a lower rate (i.e. less sensitive) during proestrus and estrus relative to metestrus and diestrus. Giamberardino et al. ${ }^{18)}$ used artificial ureteral stones as the nociceptive stimulus and quantified the time spent in behavioral crises across estrous phases. Thus, estrous cycle effects may show a difference for visceral compared to cutaneous or somatic stimuli. However, in another study, visceromotor responses to colonic distention occurred at a lower pressure during proestrus than other cycle phases. ${ }^{23)}$ These findings suggest that estrous cycle effects may vary for different visceral stimuli, and it may be that responses in reproductive structures are unique.

The estrous cycle of rats shows that both estrogen and progesterone are peak in the proestrous phase. It is difficult to analyze which hormone effects nociceptive responses, if antinociceptive effects happen. Therefore in this study, rats were ovariectomized and were implanted with a hormone pellet, so the combined effect of sex hormones was excluded.

The pain modulatory effects of experimental gonadal hormone manipulations have also been studied with mixed results. Ovariectomy decreases pain thresholds in some studies, $^{3,4)}$ but not in others. ${ }^{5)}$ Additionally, ovariectomized females demonstrated increased tail flick latencies following administration of estradiol but not progesterone, ${ }^{19)}$ but other investigators found that both estradiol and progesterone decreased tail flick latencies in ovariectomized females. $^{20)}$

In this study, nociceptive responses after acute 
inflammation was reduced by estrogen replacement. Because these results were obtained in situations of minimal restraint and by more a objective behavior test, ${ }^{12)}$ these result may suggest antinociceptive effects of estrogen.

Current views of pain processing depict a system in which neurons interact both peripherally and centrally with sensitization processes counterbalanced by inhibitory feedback systems and gonadal hormones have been demonstrated to interact with these systems at multiple levels.

The obvious first step in which gonadal hormones affect nociception is at the level of the primary afferent nerve fiber. In nonhuman animals, estrogen treatments have been demonstrated to alter receptive field properties of primary afferents in the trigeminal $^{25)}$ and pudendal nerves ${ }^{26)}$ and there is clear evidence that pregnancy and progesterone influence nerve conduction and the susceptibility of both somatic and visceral peripheral nerves to the effects of local anesthetics. ${ }^{27-31)}$ There is also evidence that response properties of silent afferents are influenced by gonadal hormones. ${ }^{32-34)}$

In addition to their effects on primary afferents, gonadal hormones clearly influence multiple central nervous system pathways involved in pain transmission. For example, sex steroids alter levels of multiple neuromodulators involved in spinal nociceptive processing, including substance $\mathrm{P}^{35-36}$ amino acids such as GABA and glutamate, and other neurotransmitters (e.g. dopamine, serotonin, and norepinephrine). ${ }^{37)}$

In addition, multiple inhibitory and excitatory systems are affected by hormonal alterations, which may alter nociceptive responses directly or indirectly. Indeed, decreased sensorimotor inhibition has been reported in healthy females during the luteal phase compared with the follicular phase of the menstrual cycle, ${ }^{38)}$ and such decreased inhibitory influence could potentiate pain transmission. Thus, hormonal effects on nociceptive processing likely result from actions at multiple peripheral and central nervous system loci.

Sex hormones also appear to influence the analgesic responses to pharmacologic agents. Several studies have demonstrated increased sensitivity to opioids among male compared to female animals. ${ }^{6-8)}$ In contrast to these findings, Ali et al. ${ }^{9)}$ showed no gender differences in responses to morphine, but gonadectomized male and female rats showed greater morphine antinociception than their intact counterparts, and ovariectomized females showed greater analgesia than gonadectomized males. Responses to nonopioid agents have also been examined, with males demonstrating greater cocaine-induced analgesia and females showing enhanced analgesic responses to nicotine. ${ }^{10)}$ Females have also demonstrated greater analgesic responses to cholinergic agonists. ${ }^{11)}$ Thus, most, but not all studies indicate enhanced opioid antinociception in male animals, while some other agents produce more robust analgesia for females.

The action mechanism of NSAIDs inhibits cyclooxygenase (COX). ${ }^{39)}$ The existence of 2 distinct isoforms of COX, which convert arachidonic acid to prostanoids, is now well known. ${ }^{40)}$ Constitutively expressed in many tissues, COX-1 produces arachidonic acid metabolites that regulate many physiologic functions under normal resting conditions, including gastric cytoprotection, platelet aggregation, vascular homeostasis and renal sodium and water balance. $^{41)}$ In contrast COX-2 is an inducible enzyme expressed in response to a variety of evoking stimuli, such as inflammatory cytokines and specific physiologic perturbations. COX-2 is upregulated at sites of inflammations, where it appears to mediate production of the bulk of arachidonic acid metabolites that are involved in inflammation and pain in certain diseases, such as arthritis. ${ }^{42-43)}$ All currently available NSAIDs inhibit both COX-1 and COX-2 at therapeutic concentrations. ${ }^{44)}$ Therefore the inhibition of COX-2 plays an important role in the analgesic and anti-inflammatory properties of NSAIDs.

In a human study of analgesic actions of NSAIDs in gender differences in pain, Walker et al. ${ }^{45)}$ showed that men had better analgesic responses to ibuprofen than women. However, the sex hormonal influences on effects of ibuprofen were not shown because the authors did not consider the menstrual cycle of the women. Little is yet known about the expression of $\mathrm{COX}$ on pain experiences 
of either naturally occurring gender differences in the relative amounts of sex steroid hormones or the effects of supplemental hormones.

In this study, the analgesic effects of ibuprofen (non-selective COX inhibitor) and NS-398 (selective COX-2 inhibitor) were investigated to elucidate indirectly the expression of COX in an acute model of arthritis in rats treated previously with sex hormones. Only NS-398 had a good analgesic effect in both the placebo and progesterone groups. It was likely that COX-2 expression was depressed by estrogen and therefore estrogen already had an antinociceptive action.

In the future it should be clinically considered that sex hormones possibly influence nociception, pain related symptoms and pharmacologic actions of analgesic drugs. Also it should be investigated more which mechanism acts on gender differences in pain perception and responses to analgesic medications with sex hormones.

In summary, it seems reasonable to conclude that nociceptive responses after acute inflammation were reduced by estrogen, and that only NS-398 had a good analgesic effect in both the placebo and progesterone groups. It is likely that the analgesic effect of NSAIDs on the estrogen group was unremarkable compared to those of the placebo and progesterone groups because of the antinociceptive action of estrogen.

\section{REFERENCES}

1. Unruh AM: Gender variations in clinical pain experience. Pain 1996; 65: 123-67.

2. Levine FM, De Simone LL: The effects of experimenter gender on pain report in male and female subjects. Pain 1991; 44: 69-72.

3. Forman LJ, Tingle V, Estilow S, Cater J: The response to analgesia testing is affected by gonadal steroids in the rat. Life Sci 1989; 45: 447-54.

4. Kepler KL, Kest B, Kiefel JM, Cooper ML, Bodnar RJ: Roles of gender, gonadectomy and estrous phase in the analgesic effects of intracerebroventricular morphine in rats. Pharmacol Biochem Behav 1989; 34: 119-27.

5. Beatty WW, Fessler RG: Gonadectomy and sensitivity to electric shock in the rat. Physiol Behav 1977; 19:
1-6.

6. Boyer JS, Morgan MM, Craft RM: Microinjection of morphine into the rostral ventromedial medulla produces greater antinociception in male compared to female rats. Brain Res 1998; 796: 315-8.

7. Cicero TJ, Nock B, Meyer ER: Gender-related differences in the antinociceptive properties of morphine. J Pharmacol Exp Ther 1996; 279: 767-73.

8. Cicero TJ, Nock B, Meyer ER: Sex-related differences in morphine's antinociceptive activity: relationship to serum and brain morphine concentrations. J Pharmacol Exp Ther 1997; 282: 939-44.

9. Ali BH, Sharif SI, Elkadi A: Sex differences and the effect of gonadectomy on morphine-induced antinociception and dependence in rats and mice. Clin Exp Pharmacol Physiol 1995; 22: 342-4.

10. Craft RM, Milholland RB: Sex differences in cocaineand nicotine- induced antinociception in the rat. Brain Res 1998; 809: 137-40.

11. Chiari A, Tobin JR, Pan H, Hood DD: Sex differences in cholinergic analgesia I. Anesthesiology 1999; 91: 1447-54.

12. Min SS, Han JS, Kim YI, Na HS, Yoon YW, Hong SK, et al: A novel method for convenient assessment of arthritic pain in voluntarily walking rats. Neurosci Lett 2001; 308: 95-8.

13. Brown-Grant K, Exley D, Naftolin F: Peripheral plasma oestradiol and luteinizing hormone concentrations during the oestrous cycle of the rat. J Endocrinol 1970; 48: $295-6$.

14. Kalra SP, Kalra PS: Temporal interrelationships among circulating levels of estradiol, progesterone and LH during the rat estrous cycle: effects of exogenous progesterone. Endocrinology 1974; 95: 1711-8.

15. Freeman ME: The ovarian cycle of the rat. The physiology of reproduction, Edited by Knobil E, Neill JD. New York. Raven Press. 1988, pp 1893-928.

16. Bradshaw HB, Temple JL, Wood E, Berkley KJ: Estrous variations in behavioral responses to vaginal and uterine distention in the rat. Pain 1999; 82: 187-97.

17. Drury RA, Gold RM: Differential effects of ovarian hormones on reactivity to electric footshock in rats. Physiol Behav 1978; 20: 187-91.

18. Giamberardino MA, Affaitati G, Valente R, Iezzi S, Vecchiet L: Changes in visceral pain reactivity as a function of estrous cycle in female rats with artificial ureteral calculosis. Brain Res 1997; 744: 234-8.

19. Martínez-Gómez M, Cruz Y, Salas M, Hudson R, 
Pacheco P: Assessing pain threshold in the rat: changes with estrus and time of day. Physiol Behav 1994; 55: 651-7.

20. Frye CA, Bock BC, Kanarek RB: Hormonal milieu affects tailflick latency in female rats and may be attenuated by access to sucrose. Physiol Behav 1992; 52: 699-706.

21. Frye CA, Cuevas CA, Kanarek RB: Diet and estrous cycle influence pain sensitivity in rats. Pharmacol Biochem Behav 1993; 45: 255-60.

22. Kayser V, Berkley KJ, Keita H, Gautron M, Guilbaud G: Estrous and sex variations in vocalization thresholds to hindpaw and tail pressure stimulation in the rat. Brain Res 1996; 742: 352-4.

23. Sapsed-Byrne S, Ma D, Ridout D, Holdcroft A: Estrous cycle phase variations in visceromotor and cardiovascular responses to colonic distension in the anesthetized rat. Brain Res 1996; 742: 10-6.

24. Leer MN, Bradbury A, Maloney JC, Stewart CN: Elevated shock threshold in sexually receptive female rats. Physiol Behav 1988; 42: 617-20.

25. Bereiter DA, Stanford LR, Barker DJ: Hormoneinduced enlargement of receptive fields in trigeminal mechano-receptive neurons. II. Possible mechanisms. Brain Res 1980; 184: 411-23.

26. Komisaruk BR, Adler NT, Hutchison J: Genital sensory field: enlargement by estrogen treatment in female rats. Science 1972; 178: 1295-8.

27. Butterworth JF, Walker FO, Lysak SZ: Pregnancy increases median nerve susceptibility to lidocaine. Anesthesiology 1990; 72: 962-5.

28. Datta S, Lambert DH, Gregus J, Gissen AJ, Covino BG: Differential sensitivities of mammalian nerve fibers during pregnancy. Anesth Analg 1983; 62: 1070-2.

29. Datta S, Migliozzi RP, Flanagan HL, Krieger NR: Chronically administered progesterone decreases halothane requirements in rabbits. Anesth Analg 1989; 68: 46-50.

30. Flanagan HL, Datta S, Lambert DH, Gissen AJ, Covino BG: Effect of pregnancy on bupivacaineinduced conduction blockade in the isolated rabbit vagus nerve. Anesth Analg 1987; 66: 123-6.

31. Kaneko M, Saito Y, Kirihara Y, Kosaka Y: Pregnancy enhances the antinociceptive effects of extradural lignocaine in the rat. Br J Anaesh 1994; 72: 657-61.

32. Berkley KJ, Robbins A, Sato Y: Afferent fibres supplying the uterus in the rat. J Neurophysiol 1988; 59: 142-63.
33. Robbins A, Sato Y, Hotta H, Berkley KJ: Responses of hypogastric nerve afferent fibers to uterine distension in estrous or metestrous rats. Neurosci Lett 1990; 110: 82-5.

34. Robbins A, Berkley KJ, Sato Y: Estrous cycle variation of afferent fibers supplying reproductive organs in the female rat. Brain Res 1992; 596: 353-6.

35. Duval P, Lenoir V, Moussaoui S, Garret C, Kerdelhué $\mathrm{B}$ : Substance $\mathrm{P}$ and neurokinin A variations throughout the rat estrous cycle; comparison with ovariectomized and male rats: II. Trigeminal nucleus and cervical spinal cord. J Neurosci Res 1996; 45: 610-6.

36. Kerdelhué B, Tartar A, Lenoir V, Abed AE, Hublau P, Millar RP: Binding studies of substance $P$ anterior pituitary binding sites: changes in substance $\mathrm{P}$ binding sites during the rat estrous cycle. Regul Pept 1985; 10: 133-43.

37. Smith SS: Female sex steroid hormones: from receptors to networks to performance-actions on the sensorimotor system. Prog Neurobiol 1994; 44: 55-86.

38. Swerdlow NR, Hartman PL, Auerbach PP: Changes in sensorimotor inhibition across the menstrual cycle: implications for neuropsychiatric disorders. Biol Psychiatry 1997; 41: 452-60.

39. Moskowitz RW: The appropriate use of NSAIDs in arthritic conditions. Am J Orthop 1996; 25 Suppl. 9: 4-6.

40. Smith WL, Garavito RM, DeWitt DL: Prostaglandin endoperoxide $\mathrm{H}$ synthases (cyclooxygenases)-1 and -2 . J Biol Chem 1996; 271: 33157-60.

41. Zhang Y, Shaffer A, Portanova J, Seibert K, Isakson PC: Inhibition of cyclooxygenase-2 rapidly reverses inflammatory hyperalgesia and prostaglandin E2 production. J Pharmacol Exp Ther 1997; 283: 1069-75.

42. Cashman JN: The mechanisms of action of NSAIDs in analgesia. Drugs 1996; 52 Suppl. 5: 13-23.

43. Beiche F, Scheuerer S, Brune K, Geisslinger Gerd, Goppelt-Struebe M: Up-regulation of cyclooxygenase-2 mRNA in the rat spinal cord following peripheral inflammation. FEBS Lett 1996; 390: 165-9.

44. Cryer B, Feldman M: Cyclooxygenase-1 and cyclooxygenase-2 selectivity of widely used nonsteroidal anti-inflammatory drugs. Am J Med 1998; 104: 41321.

45. Walker JS, Carmody JJ: Experimental pain in healthy human subjects: gender differences in nociception and in response to ibuprofen. Anesth Analg 1998; 86: $1257-62$. 